\title{
What is the optimal body mass index for Chinese people?
}

\author{
Shankuan Zhu MD PhD, Xiaoguang Ma MD MPhil, Jin-Ling Tang MD PhD
}

See related research article by Lin and colleagues at www.cmaj.ca/cgi/doi/10.1503/cmaj.100303.

I $\mathrm{n}$ this issue, Lin and colleagues report the findings of their study of the association between body mass index (BMI) and allcause mortality among Chinese adults aged 20 years or older. ${ }^{1}$ Using data collected over 10 years for 124456 participants, they observed a U-shaped association between BMI and mortality. A BMI of 24.0-25.9 for men and women was associated with the lowest mortality and was deemed by the authors as optimal.

Lin and colleagues' findings are consistent with those from previous studies in Chinese and white populations. In a cohort study involving 169871 Chinese people aged 40 years or older, for example, $\mathrm{Gu}$ and colleagues found that a BMI of 24.0-24.9 was related to the lowest risk of death. ${ }^{2}$ In white populations, extremely low and high BMI values were found to be associated with an excess risk of death in several nationwide studies..$^{3-5}$ In a more recent study involving 11326 Canadians aged 25 years or older, Orpana and colleagues found that both underweight $(\mathrm{BMI}<18.5)$ and severe obesity $(\mathrm{BMI} \geq 35)$ were strong risk factors for all-cause mortality. ${ }^{4}$ However, the optimal BMI values reported in the two Chinese studies ${ }^{1,2}$ were higher than those reported in the white populations. For example, on the basis of data from 19 prospective studies involving a total of 1.46 million white adults, Berrington de Gonzalez and colleagues found that BMI values of 22.5-24.9 were optimal in white adult populations. ${ }^{5}$

The findings in the study by Lin and colleagues are important because they seem to challenge the current definitions of overweight and obesity and are inconsistent with the pattern found in white populations and for the relation between BMI and chronic diseases, in particular cardiovascular diseases. The World Health Organization (WHO) defines overweight as a BMI of 25 or higher and obesity as a BMI of 30 or higher. Lower BMI cutoff values for overweight and obesity have been suggested for Chinese populations by WHO and others. ${ }^{6}$ For example, Zhou suggested cutoff values of 24 for overweight and 28 for obesity among Chinese adults. ${ }^{7}$ Weng and colleagues reported that a BMI of 23 seemed more appropriate for identifying Chinese people at increased risk of obesity-related metabolic disorders. ${ }^{8}$ However, according to the findings by Lin and colleagues ${ }^{1}$ and by $\mathrm{Gu}$ and colleagues, ${ }^{2}$ these BMI values used to define overweight fall clearly in the range of optimal values. Is there sufficient evidence to consider raising the BMI cutoff for overweight and obesity in the adult Chinese population?

Over the past decades, the prevalence of cardiovascular diseases and diabetes in China has climbed to a level almost equal to that in developed countries. The prevalence of overweight and obesity, although still lower than that in developed countries, is increasing rapidly. For example, from 1992 to 2002, the prevalence of overweight in China increased by $38.6 \%$, and the prevalence of obesity by $80.6 \% .^{9}$ In 2002 , the average BMI among Chinese adults was re ported to be a few units lower than that among white adults. ${ }^{10}$ In addition, Asian people are reported to have a higher percentage of body fat and more abdominal fat than white people with the same BMI. ${ }^{11}$ Central obesity defined by such methods as waist circumference has been shown to be more strongly related to the risk of cardiovascular diseases and other chronic diseases than obesity defined by BMI. Consequently, a lower BMI value may still be associated with an increased risk of chronic diseases, especially cardiovascular disease and diabetes. These observations all suggest that the optimal BMI values in the adult Chinese population should be lower than those in the white population, which contradicts what is suggested by Lin and colleagues.
Competing interests: None declared.

This article was solicited and has not been peer reviewed.

Correspondence to:

Dr. Shankuan Zhu, zsk@zju.edu.cn

CMAJ 2011. DOI:10.1503 /cmaj.110142 
The critical part of the curve for determining whether BMI values of 24.0-25.9 are optimal is the lower end rather than the upper end we have discussed above. If the lower BMI values associated with increased mortality are not causal or only partially causal, the upward tail of the curve at the low end would be "pressed down" and the association between BMI and mortality would become a linear line or a J-shaped curve instead of a U-shaped curve. Either a J-shaped curve or a linear line would still suggest that the current or an even lower BMI would be appropriate for defining overweight and obesity.

Can the increased mortality among people with very low BMI values be explained by factors other than a low BMI? Evidence seems to suggest that the increased mortality among underweight people may be due at least in part to reverse causality, ${ }^{12}$ whereby a chronic, undiagnosed disease develops first, and the patient later loses weight as a result. In this scenario, both death and low BMI are the consequences of the chronic disease; low BMI will be associated with, but is not the true cause of, the excess mortality. Lin and colleagues did reanalyze their data by excluding people who died during the first three years of follow-up, but they did not report the results in detail.

But is three years long enough to exclude most people in whom an asymptomatic disease such as cancer has already developed before the study has begun? Freedman and colleagues found that the risk of death among people with low BMI values was reduced substantially when they excluded smokers and people who died during the first five years of follow-up..$^{12}$ Berrington de Gonzalez and colleagues found that the hazard ratios for a BMI below 20.0 were attenuated with longer-term follow-up. ${ }^{5}$

In conclusion, the study by Lin and colleagues carries an important message about the appropriateness of the current definition of overweight and obesity. Their findings suggest a BMI for Chinese adults that is higher than the currently recommended cutoff value for defining overweight and obesity in Chinese populations. However, raising the cutoff could play down the cur- rent burden of obesity and lead to further increases in its related diseases. More evidence is needed to clarify whether a low BMI is a direct cause of mortality or merely a consequence of pre-existing chronic diseases that cause death.

\section{References}

1. Lin WC, Tsai SL, Albu J, et al. Body mass index and allcause mortality in a large Chinese cohort. CMAJ 2011;183: E329-36.

2. Gu D, He J, Duan X, et al. Body weight and mortality among men and women in China. JAMA 2006;295:776-83.

3. Flegal KM, Graubard BI, Williamson DF, et al. Excess deaths associated with underweight, overweight, and obesity. JAMA 2005; 293:1861-7.

4. Orpana HM, Berthelot JM, Kaplan MS, et al. BMI and mortality: results from a national longitudinal study of Canadian adults. Obesity (Silver Spring) 2010;18:214-8.

5. Berrington de Gonzalez A, Hartge P, Cerhan JR, et al. Bodymass index and mortality among 1.46 million white adults. $N$ Engl J Med 2010;363:2211-9.

6. The Asia-Pacific perspective: redefining obesity and its treatment. World Health Organization/International Association for the Study of Obesity/International Obesity Task Force; 2000. Available: www.wpro.who.int/NR/rdonlyres/0A35147B-B1D5-45A6 -9FF2-F7D86608A4DE/0/Redefiningobesity.pdf (accessed 2011 Feb. 22).

7. Zhou B. Predictive values of body mass index and waist circumference to risk factors of related diseases in Chinese adult population [article in Chinese]. Zhonghua Liu Xing Bing Xue Za Zhi 2002;23:5-10.

8. Weng X, Liu Y, Ma J, et al. Use of body mass index to identify obesity-related metabolic disorders in the Chinese population. Eur J Clin Nutr 2006;60:931-7.

9. Ma GS, Li YP, Wu YF, et al. The prevalence of body overweight and obesity and its changes among Chinese people during 1992 to 2002 [article in Chinese]. Zhonghua Yu Fang Yi Xue Za Zhi 2005;39:311-5.

10. Chinese Medical Association Subsection of Cardiovascular Disease, Chinese Journal of Cardiology Editorial Board. Highlights of the Second National Conference on Dyslipidemia. Chin J Cardiol 2002;30:643-6.

11. Wang J, Thornton JC, Russell M, et al. Asians have lower body mass index (BMI) but higher percent body fat than do whites: comparisons of anthropometric measurements. Am J Clin Nutr 1994;60:23-8.

12. Freedman DM, Ron E, Ballard-Barbash R, et al. Body mass index and all-cause mortality in a nationwide US cohort. Int $J$ Obes (Lond) 2006;30:822-9.

Affiliations: From the Obesity and Body Composition Research Center (Zhu), School of Public Health, Zhejiang University, Hangzhou, China; the Department of Epidemiology and Biostatistics (Ma), Arnold School of Public Health, University of South Carolina, Columbia, SC; and School of Public Health and Primary Care (Tang), the Chinese University of Hong Kong, Hong Kong, China

Contributors: All of the authors contributed substantially to the writing and revising of the manuscript and approved the final version submitted for publication. 